\title{
Simultaneous multiple well-seismic ties using flattened synthetic and real seismograms
}

\author{
Xinming $\mathrm{Wu}^{1}$ and Guillaume Caumon ${ }^{2}$
}

\begin{abstract}
Well-seismic ties allow rock properties measured at well locations to be compared with seismic data and are therefore useful for seismic interpretation. Numerous methods have been proposed to compute well-seismic ties by correlating real seismograms with synthetic seismograms computed from velocity and density logs. However, most methods tie multiple wells to seismic data one by one; hence, they do not guarantee lateral consistency among multiple well ties. We therefore propose a method to simultaneously tie multiple wells to seismic data. In this method, we first flatten synthetic and corresponding real seismograms so that all seismic reflectors are horizontally aligned. By doing this, we turn multiple wellseismic tying into a $1 \mathrm{D}$ correlation problem. We then compute only vertically variant but laterally constant shifts to correlate these horizontally aligned (flattened) synthetic and real seismograms. This two-step correlation method maintains lateral consistency among multiple well ties by computing a laterally and vertically optimized correlation of all synthetic and real seismograms. We applied our method to a 3D real seismic image with multiple wells and obtained laterally consistent well-seismic ties.
\end{abstract}

\section{INTRODUCTION}

Well logs, such as the velocity logs in Figure 1a, are often used to calibrate seismic interpretation because they provide more accurate information of the subsurface at well locations. A seismic image of geologic structures, such as the one in Figure 1a, can be used to extend the locally measured well-log properties to obtain a global interpretation of the subsurface (Fomel, 2010; Hale, 2010b). Such a joint interpretation of seismic and well-log data, however, first requires tying well logs measured in depth to the seismic data in two-way time. For the example in Figure 1b, we directly extrapolate the untied velocity-log values along seismic reflections, and we obtain an inaccurate $3 \mathrm{D}$ velocity model that displays geologically unrealistic discontinuities (highlighted by the red arrows in Figure 1b) along reflectors.

Many authors (White, 1997; White and Simm, 2003; Herrera and van der Baan, 2014) have discussed the common steps of well-seismic ties, which often include (1) estimating an approximate timedepth function to convert the wells to the time domain, (2) choosing an appropriate wavelet, (3) constructing synthetic seismograms, and (4) matching them with corresponding real seismic data. For the first step, the time-depth function is typically approximated using check-shot data or by integrating the sonic log. For the second step, Muñoz and Hale (2015) choose a Ricker wavelet with the peak frequency of the seismic image. A more appropriate wavelet may be extracted from real seismograms close to well locations (White, 1980; Ziolkowski et al., 1998). For the third step, most methods (White and Simm, 2003; Herrera and van der Baan, 2014; Herrera et al., 2014) construct a synthetic seismogram by convolving the wavelet with the reflectivity series calculated from velocity and density logs. Alternatively, Muñoz and Hale (2015) use a propagator matrix method to compute more realistic synthetic seismograms with multiples, attenuations, and dispersion.

Most automatic well-seismic tying methods focus on the last step to find vertical shifts that match a well log with the seismic image. These shifts are often nonlinear (Herrera and van der Baan, 2014; Herrera et al., 2014) and may vary rapidly, which makes some windowed crosscorrelation methods (White, 1980; White and Simm, 2003) fail when the shifts vary within a chosen window size. Therefore, some authors (Herrera and van der Baan, 2014; Herrera et al., 2014; Cheverry et al., 2015; Muñoz and Hale, 2015) propose to use dynamic time warping (DTW) or equivalent methods to better match the synthetic seismogram with the real seismogram. DTW was first proposed by Sakoe and Chiba (1978) in the context of speech recognition, and today it is widely used in geophysics for correlating

Manuscript received by the Editor 2 June 2016; revised manuscript received 15 August 2016; published online 09 November 2016.

${ }^{1}$ Colorado School of Mines, Golden, Colorado, USA. E-mail: xinwucwp@gmail.com.

${ }^{2}$ Colorado School of Mines, Golden, Colorado, USA and Université de Lorraine, GeoRessources, ENSG, Vandoeuvre-Lés-Nancy, France. E-mail: guillaume. caumon@univ-lorraine.fr.

(C) 2017 Society of Exploration Geophysicists. All rights reserved. 
seismic traces (Anderson and Gaby, 1983), seismic images (Hale, 2013), well logs (Smith and Waterman, 1980; Wheeler and Hale, 2014), and magnetostratigraphic data (Lallier et al., 2013). Compared with windowed crosscorrelation methods, DTW is often more accurate in computing relative shifts between series or images, especially when the shifts are large and rapidly varying.

Although many methods have been proposed for all three steps of well-seismic ties, most of them (White, 1980; White and Simm, 2003; Herrera and van der Baan, 2014; Herrera et al., 2014) tie multiple wells to the seismic image one by one and often have difficulties in maintaining lateral consistency among multiple wells. To simultaneously tie multiple wells to a seismic image, Muñoz and Hale $(2014,2015)$ first use multiple synthetic seismograms, computed from the wells, to interpolate a synthetic amplitude image. Because the interpolation is guided by the real seismic image as discussed by Hale (2010b), the structures of the interpolated synthetic image laterally conform to the real seismic image. They then match the synthetic image with the real seismic image using smooth dynamic image warping (Compton and Hale, 2014). Cubizolle et al. (2015) propose a similar method to compute simultaneous multiple well ties, but they use a relative geologic-time (RGT) volume (Stark, 2004; Wu and Zhong, 2012), computed from the real seismic image, to interpolate a similar synthetic image. These methods often produce robust and laterally consistent multiple well ties. However, they require constructing a synthetic image with an image-guided interpolation or RGT volume. Computing such an interpolation or RGT volume is computationally expensive and is sensitive to the presence of faults and unconformities. In addition, one potential problem of these methods is that the interpolated initial synthetic image is often laterally inconsistent (e.g., as shown in Figure 1b) because wells are initially misaligned with the seismic image.

To address this problem, we propose to first laterally correlate synthetic seismograms and the corresponding real seismograms to obtain flattened synthetic and real seismograms, in both of which seismic events corresponding to the same layers are horizontally aligned. We then vertically match the flattened synthetic seismograms with the flattened real ones using only vertically variant but laterally invariant shifts, which is a 1D correlation problem. With this two-step correlation method, we are able to efficiently compute simultaneous multiple well-seismic ties and effectively maintain lateral consistency among multiple wells because the events corresponding to the same layers in the synthetic seismograms are correlated with those corresponding to the same layers in the real seismograms.

The paper is organized as follows: We first briefly discuss computation of synthetic seismograms, the DTW method, and single well-seismic ties. We then discuss in detail how to compute simultaneous multiple well-seismic ties using our two-step correlation method. We finally demonstrate the results of well-seismic ties by interpolating a 3D velocity model from the tied velocity logs using an image-guided interpolation method (Hale, 2010b).

\section{TYING WELLS INDEPENDENTLY}

In tying a single well to a seismic image, we begin by choosing a wavelet, computing a reflectivity series in depth, and generating an initial time-depth function. We then generate an initial synthetic seismogram by convolving the reflectivity series with the wavelet delayed by the initial time-depth function. We then use smooth DTW (Compton and Hale, 2014) to compute vertical shifts that match the synthetic seismogram with the corresponding real seismogram, and we use these shifts to update the time-depth function. We iteratively update the time-depth function and the synthetic seismogram until the updates are insignificant to finally obtain the single well-seismic tie, as is described by Muñoz and Hale (2015).

\section{Synthetic seismograms}

The 3D seismic image and well logs that we use in this paper are from the freely available Teapot Dome data set (Anderson, 2009). In this paper, we choose only the well logs with velocity and density logs that are significantly long in the same depth ranges, as shown in Figure 1a.

From these velocity $v(z)$ (Figure 1a) and corresponding density $\rho(z)$ (not shown) logs, the reflectivity series can be computed as follows by assuming a layered-earth model (Sheriff and Geldart, 1995):

$$
r(z)=\frac{v(z+\Delta z) \rho(z+\Delta z)-v(z) \rho(z)}{v(z+\Delta z) \rho(z+\Delta z)+v(z) \rho(z)},
$$

where the depth sampling interval in this case is $\Delta z=0.1524 \mathrm{~m}$. An initial time-depth function for each well can be estimated from the velocity $\log$ of that well a)

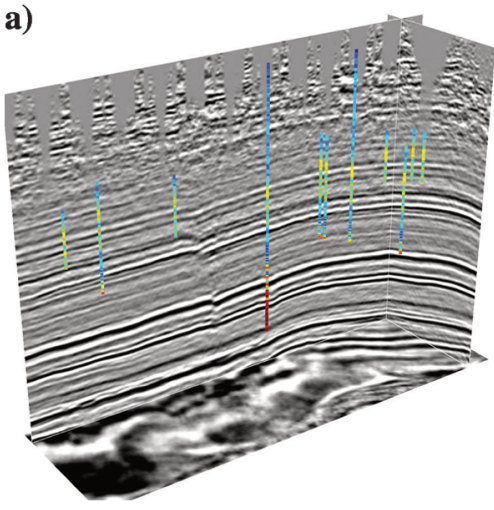

b)

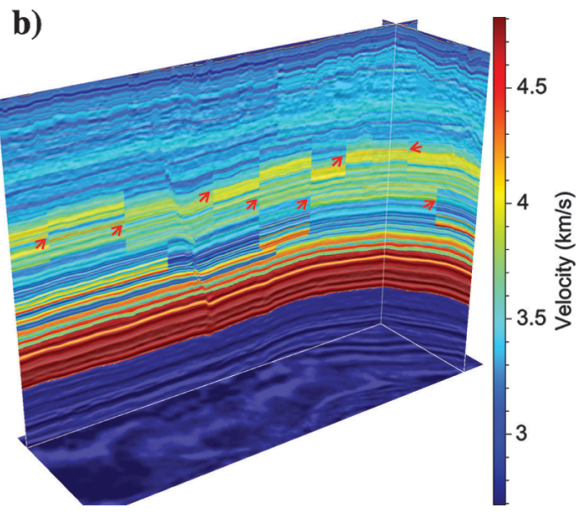

Figure 1. A seismic image is displayed with 11 velocity logs in (a). These velocity logs are displayed in time using the initial time-depth functions. A 3D image-guided nearest neighbor interpolation (b) of these velocity logs is laterally discontinuous (highlighted by red arrows) along reflectors because the logs are not tied to the seismic image.

$$
\tau_{0}(z)=\tau_{\min }+2 \int_{z_{\min }}^{z} \frac{d \xi}{v(\xi)},
$$

where $\tau_{\min }=2 z_{\min } / v\left(z_{\min }\right)$.

The wavelet we choose for this example is a zero-phase Ricker wavelet $w(\tau)$ with $35 \mathrm{~Hz}$ peak frequency, which is also the peak frequency of the real seismic data. A more accurate wavelet might be estimated from nearby seismic traces by using a coherency matching technique (White, 1980; Walden and White, 1998) or by rotating and shifting a given zero-phase wavelet so that it best matches the energy spectrum of the seismic data (White and Simm, 2003). For multiple well-seismic ties, it might be desirable to account for spatial and temporal changes in seismic wavelets between wells as discussed by Ziolkowski et al. (1998). However, we use a Ricker wavelet in this 
paper to avoid potential uncertainties due to the wavelet estimation and also to demonstrate that our method is robust even using a single simple wavelet for constructing all the synthetic seismograms.

After choosing a zero-phase Ricker wavelet, we then construct a synthetic seismogram for each well by convolving the reflectivity series $(r(z))$ with the wavelet $(w(\tau))$ delayed by the initial timedepth function $\left(\tau_{0}(z)\right)$ :

$$
f(\tau)=\int_{z_{\min }}^{z_{\max }} r(z) w\left(\tau-\tau_{0}(z)\right) d z .
$$

Using this simple convolution method, we compute all the synthetic seismograms that are denoted by red and overlaid with the real seismograms (black) in Figure 2a. These real seismograms are directly extracted from the seismic image at locations closest to the wells. The synthetic seismograms computed in this way are all zero phase, which is likely inconsistent with the corresponding real seismic data. Therefore, we estimate a constant-phase rotation to these synthetic seismograms using a DTW method described by Muñoz and Hale (2012). As shown in Figure 2a, these synthetic seismograms (red), computed with the initial time-depth functions (equation 2), do not match the real seismograms (black). Next, we will discuss how to update the time-depth functions one by one for better single well-seismic ties using smooth DTW (Compton and Hale, 2014).

\section{DTW}

To match a synthetic seismogram $f(\tau)$ with a real seismogram $g(t)$, we expect to shift the former by $s(\tau)$ so that they are approximately the same: $f(\tau+s(\tau)) \approx g(t(\tau))$. The shifts $s(\tau)$ are often large and nonlinear (Herrera and van der Baan, 2014) and therefore are difficult to estimate using windowed crosscorrelation methods. The DTW method, first proposed by Sakoe and Chiba (1978) in speech recognition, is a better method to estimate nonlinear and rapidly varying shifts (Hale, 2013; Herrera and van der Baan, 2014; Muñoz and Hale, 2015). DTW corresponds in solving the following constrained optimization problem:

$$
\begin{gathered}
\min _{s(\tau)} \sum_{\tau}\|g(t(\tau))-f(\tau+s(\tau))\|_{p} \\
\text { subject to } \varepsilon_{\min } \leq \frac{d s}{d \tau} \leq \varepsilon_{\max },
\end{gathered}
$$

compute coarsely sampled shifts and then interpolate back smooth shifts for all samples.

After computing the vertical shifts $s(\tau)$ that match the synthetic seismogram with the real seismogram, we can then compute an updated time-depth function $\tau_{1}(z)$ from a given initial time-depth function $\tau_{0}(z)$ :

$$
\tau_{1}(z)=\tau_{0}(z)+s\left(\tau_{0}(z)\right)
$$

These shifts $s(\tau)$ correspond to squeezing and stretching of the synthetic seismograms in matching the seismic traces, and such squeezing and stretching can be unreasonably excessive as discussed by White and Simm (2003). To avoid such excessive stretching and squeezing, the shifts are constrained to satisfy some specified bounds on the time derivatives $d s / d \tau$ of the shifts in the smooth DTW method (equation 4). As suggested by Muñoz and Hale (2015), we can relate the time derivatives $d s / d \tau$ to the initial and updated velocity functions $v_{0}(z)=2 d z / d \tau_{0}$ and $v_{1}(z)=2 d z / d \tau_{1}$, respectively. From equation 5, we have

$$
\frac{d \tau_{1}}{d z}=\frac{d \tau_{0}}{d z}+\frac{d s}{d \tau_{0}} \frac{d \tau_{0}}{d z},
$$

which is equivalent to

$$
\frac{d s}{d \tau_{0}}=\frac{v_{0}(z)}{v_{1}(z)}-1
$$

This means that we can specify bounds on $v_{0}(z) / v_{1}(z)$ to impose corresponding constraints on the shifts (Muñoz and Hale, 2015):

$$
\left(\frac{v_{0}(z)}{v_{1}(z)}\right)_{\min }-1 \leq \frac{d s}{d \tau_{0}} \leq\left(\frac{v_{0}(z)}{v_{1}(z)}\right)_{\text {max }}-1 .
$$

The updated velocity $v_{1}(z)$ should have a similar trend as the initial velocity $v_{0}(z)$ (measured well-log velocity), which provides a qualitative way to determine the bounds on the velocity ratio $v_{0}(z) / v_{1}(z)$ by qualitatively evaluating the reasonableness of updated velocity comparing with the initial velocity (Muñoz and Hale, 2015). In this way, we are seeking for shifts that not only optimally match the synthetic and real seismograms but also reasonably update the where $p>0$. In this paper, we choose $p=2$ corresponding to the $L_{2}$ norm for examples. We will discuss how to specify the constraint bounds of $d s / d \tau$ in computing the shifts.

One potential problem of the common DTW method (Hale, 2013; Herrera and van der Baan, 2014; Herrera et al., 2014) is that the estimated shifts are limited to integers, which may not be sufficient to accurately correlate the synthetic and real seismograms. We use the smooth DTW method, proposed by Compton and Hale (2014), to compute smoothly varying shifts, which are often more accurate than those from the common DTW method, as suggested by Muñoz and Hale (2015). As described in detail by Compton and Hale (2014), the smooth DTW method solves the same minimization problem (equation 4) to first
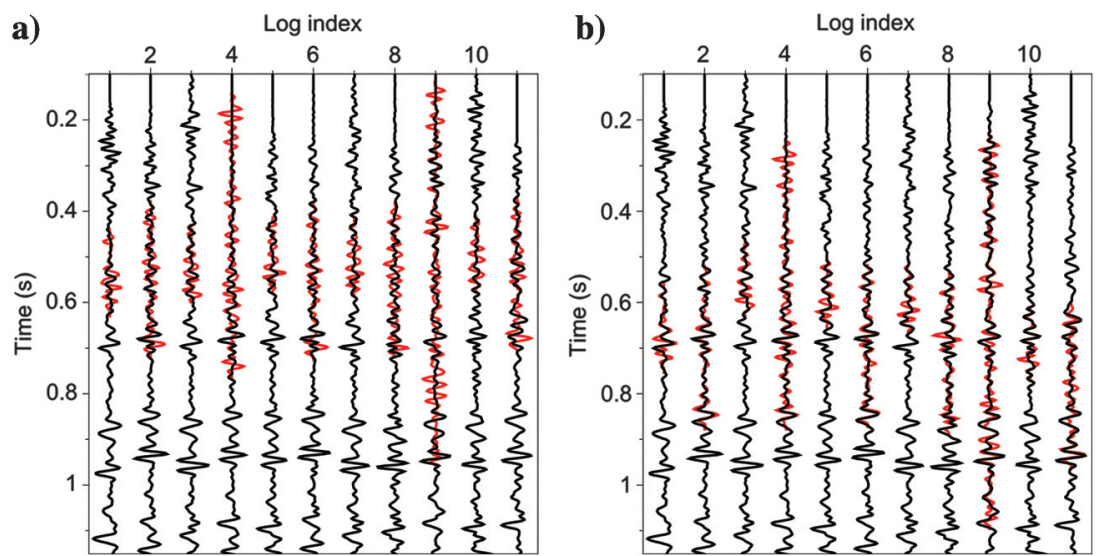

Figure 2. Initial (a) synthetic seismograms (red) are independently aligned (b) with real seismograms (black). 
velocity. In all examples in this paper, we choose $\left(v_{0}(z) / v_{1}(z)\right)_{\min }=$ 0.9 and $\left(v_{0}(z) / v_{1}(z)\right)_{\max }=1.2$.

Errors and uncertainties may exist in computing the initial timedepth functions for constructing synthetic seismograms. Therefore, the updated time-depth function (equation 5) should be used to compute a new synthetic seismogram that should be again correlated with the real seismogram to compute new possible shifts. This means that the whole process should be iteratively performed until updates become negligible and a stable calibrated time-depth function is obtained.

Using this iterative process, we update the time-depth functions one by one and independently match the synthetic (red) seismograms with the real (black) seismograms (Figure 2b) one by one. We observe that the synthetic seismograms vertically match the real seismograms, which indicates that the smooth DTW method successfully finds the optimal vertical match for each pair of synthetic and real seismograms independently. However, an optimal match of a synthetic seismogram and the corresponding real seismogram does not necessarily guarantee a reliable well-seismic tie because errors and noise may exist in synthetic and real seismograms.

Using the updated time-depth function computed for each well, we can independently tie the velocity logs to the seismic image as shown in Figure 3a. One way to check for possible errors in wellseismic ties is to extend the well-log measurements along seismic reflectors to compute an image-guided nearest neighbor interpolation (Hale, 2010b) of the measurements. In this method, assuming that we have a set of $k$ known well-log values $\mathcal{V}=\left\{v_{1}, v_{2}, \ldots, v_{k}\right\}$ $\left(v_{k} \in \mathbb{R}\right)$ that are spatially scattered at corresponding $k$ known locations $\mathcal{X}=\left\{\mathbf{x}_{1}, \mathbf{x}_{2}, \ldots, \mathbf{x}_{k}\right\}$, we then compute an image-guided nearest neighbor interpolation of the known values by solving the following anisotropic eikonal equation (Hale, 2009):

$$
\begin{array}{cc}
\nabla t(\mathbf{x}) \cdot \mathbf{D}(\mathbf{x}) \nabla t(\mathbf{x})=1, & \mathbf{x} \notin \mathcal{X} \\
t(\mathbf{x})=0, & \mathbf{x} \in \mathcal{X},
\end{array}
$$

where $\mathbf{x}=\left(x_{1}, x_{2}, x_{3}\right)$ represent the $3 \mathrm{D}$ spatial coordinates within the 3D seismic image (Figure 1a) and $t(\mathbf{x})$ is a map of non-Euclidean distance (Hale, 2009) from $\mathbf{x}$ to the nearest known sample $\mathbf{x}_{k}$. When solving the above anisotropic eikonal equation for the minimal-distance map $t(\mathbf{x})$, it is straightforward to simultaneously ob-

a)

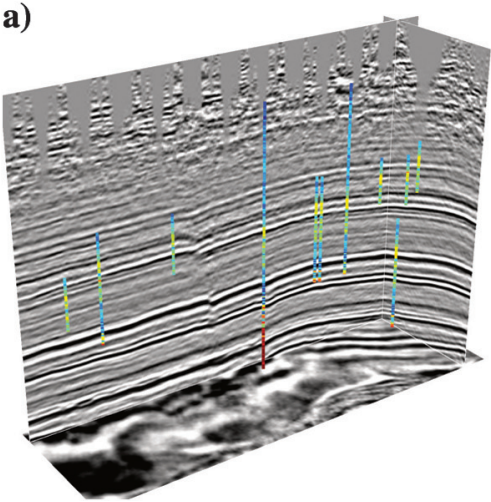

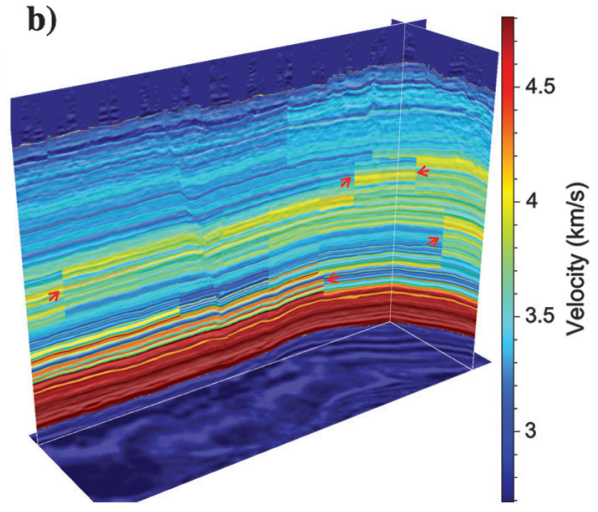

b)
Figure 3. Velocity logs are independently tied (a) to the seismic image using time-depth functions computed from single well-seismic ties. A 3D image-guided nearest neighbor interpolation (b) of these logs is laterally more continuous than the one in Figure $1 \mathrm{~b}$, but it is still discontinuous at some positions (highlighted by red arrows), which indicates that some logs may not be correctly tied to the seismic image. tain the nearest neighbor interpolant (Hale, 2009). A known sample $\mathbf{x}_{k}$ is nearest to a point $\mathbf{x}$ only if the non-Euclidean distance $t(\mathbf{x})$ is less than that for any other known sample point. The metric tensor field $\mathbf{D}(\mathbf{x})$ represents the coherence and orientation of seismic structures and therefore often provides anisotropic and spatially variant coefficients for the eikonal equation. As discussed by Hale (2010a), we construct the metric tensor field using structure tensors (Van Vliet and Verbeek, 1995; Fehmers and Höcker, 2003) computed from the $3 \mathrm{D}$ seismic image. With such a metric tensor field, the computed nearest neighbor interpolant conforms to structures apparent in the seismic image.

If the well logs are correctly aligned with the seismic image, the image-guided nearest neighbor interpolation of well-log measurements should be laterally continuous along the seismic reflectors. We observe that the interpolation (Figure $3 \mathrm{~b}$ ) of the velocities after single well-seismic ties (Figure 3a) is laterally more consistent than the one before tying (Figure 1b). However, some lateral discontinuities (highlighted by red arrows in Figure 3b) are still apparent in the interpolant, and these discontinuities do not coincide with geologic faults. This indicates that errors exist in the single wellseismic ties without any lateral control. Next, we will discuss how to efficiently compute simultaneous multiple well-seismic ties to improve the lateral consistency among these multiple wells.

\section{TYING WELLS SIMULTANEOUSLY}

The idea of tying multiple wells simultaneously is proposed by Muñoz and Hale (2015) and Cubizolle et al. (2015), who suggest to first extrapolate the synthetic seismograms away from well locations, following the real seismic reflections, to compute a synthetic image. Then, they match the synthetic image with the corresponding real seismic image to compute simultaneous multiple well-seismic ties. However, constructing a 2D or 3D synthetic image and matching it with the real seismic image significantly increases the computational cost. In addition, the initial synthetic image, interpolated with uncorrelated synthetic seismograms (or well logs), is often laterally discontinuous, like the interpolated velocity image shown in Figure 1b.

We propose to first compute laterally correlated (flattened) synthetic and real seismograms, in both of which all events corresponding to the same geologic layers are horizontally aligned. We then match the flattened synthetic seismograms with the flattened real seismograms using only vertically variant but laterally invariant shifts, which is a $1 \mathrm{D}$ correlation problem.

\section{Flattening synthetic and real seismo- grams}

We use the method proposed by Wheeler and Hale (2014) for simultaneously flattening multiple well $\operatorname{logs}$ to flatten synthetic and real seismograms and obtain horizontally aligned seismograms, as shown in Figures 4 and 5. Like all seismic chronostratigraphic methods, this essentially assumes that there is limited lateral variation of reflectivity along the isochron geologic horizons. We will further discuss the hypotheses underlying our method in the "Discussion" section. 
Let us denote the synthetic seismograms (Figure 4a) as $f(I, \tau)$, where $I \in\{1, \ldots, 11\}$ represents the index of the seismograms. In flattening the synthetic seismograms, we first apply the DTW method to all possible pairs $\left(N_{p}=(11(11-1)) / 2=55\right)$ of the seismograms and find the optimal correlation for each pair $f(K, \tau)$ and $f(J, \tau)$, where $K, J \in\{1, \ldots, 11\}$ and $K \neq J$. This pairwise correlation locates the samples $\tau_{k}$ and $\tau_{j}$, which correspond to the same geologic layer or geologic time in the seismograms $K$ and $J$, respectively. We then compute shifts $s_{f}\left(K, \tau_{k}\right)$ and $s_{f}\left(J, \tau_{j}\right)$ that shift the samples $\tau_{k}$ and $\tau_{j}$, corresponding to the same geologic layer, to the same RGT (Wheeler and Hale, 2014),

$$
\begin{aligned}
u_{f}\left(K, \tau_{k}\right) & =\tau_{k}+s_{f}\left(K, \tau_{k}\right)=u_{f}\left(J, \tau_{j}\right) \\
& =\tau_{j}+s_{f}\left(J, \tau_{j}\right)
\end{aligned}
$$

where $u_{f}$ represent RGT and $u_{f}\left(K, \tau_{k}\right)=$ $u_{f}\left(J, \tau_{j}\right)$ because the samples $\tau_{k}$ and $\tau_{j}$ belong to the same geologic layer according to the pairwise correlation of the seismograms $K$ and $J$. In this equation, the sample positions $\tau_{k}$ and $\tau_{j}$ are knowns that are located by the pairwise correlation, whereas $s_{f}\left(K, \tau_{k}\right)$ and $s_{f}\left(J, \tau_{j}\right)$ are unknowns.

As discussed in detail by Wheeler and Hale (2014), we define equation 10 for all samples in all the 55 pairs of the synthetic seismograms. We then simultaneously solve these equations in the least-squares sense to compute shifts $s_{f}(I, \tau)$ that map all the seismograms $f(I, \tau)$ into RGT and obtain the flattened seismograms $f\left(I, u_{f}\right)$ as shown in Figure $4 \mathrm{~b}$. The shifts $s_{f}(I, \tau)$ are denoted as a $2 \mathrm{D}$ function because they vary vertically with $\tau$ and laterally with the seismogram index $I$. Note that the mapping $u_{f}(I, \tau)=$ $\tau+s_{f}(I, \tau)$ is a monotonic function between $\tau$ and $u_{f}$ for each seismogram $I$, which means that we can map the flattened seismograms (Figure $4 \mathrm{~b}$ ) back to obtain the original seismograms (Figure 4a).

Similarly, we can also compute flattened (Figure $5 \mathrm{~b})$ real seismograms $g\left(I, u_{g}\right)$ in RGT $u_{g}$ from the original (Figure 5a) traces $g(I, t)$ in two-way time $t$. The mapping between $u_{g}$ and $t$ is computed by $u_{g}(I, t)=t+s_{g}(I, t)$, where the shifts $s_{g}(I, t)$ for the real seismograms are also computed using the method described above. As shown in Figures $4 \mathrm{~b}$ and $5 \mathrm{~b}$, events in the flattened synthetic and real seismograms are horizontally aligned. Our next step is to vertically shift the flattened synthetic seismograms (Figure 4b) to match them with the flattened real seismograms (Figure 5b).

\section{Matching flattened seismograms}

As shown in Figure 6a, the flattened synthetic seismograms (red) are not vertically aligned with tening. the flattened real seismograms (black); however, events in both are horizontally aligned. Therefore, we can obtain an optimal match between the flattened synthetic seismograms $f\left(I, u_{f}\right)$ and the real seismograms $g\left(I, u_{g}\right)$ with only vertically variant but laterally invariant shifts $s_{u}\left(u_{f}\right)$ that can be computed by solving the following minimization problem:
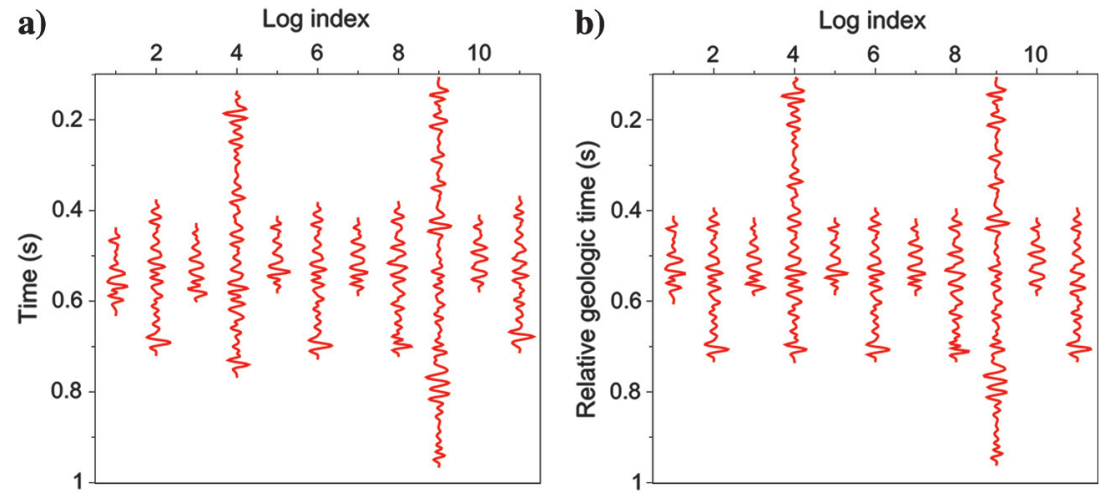

Figure 4. Synthetic seismograms (a) before and (b) after flattening.
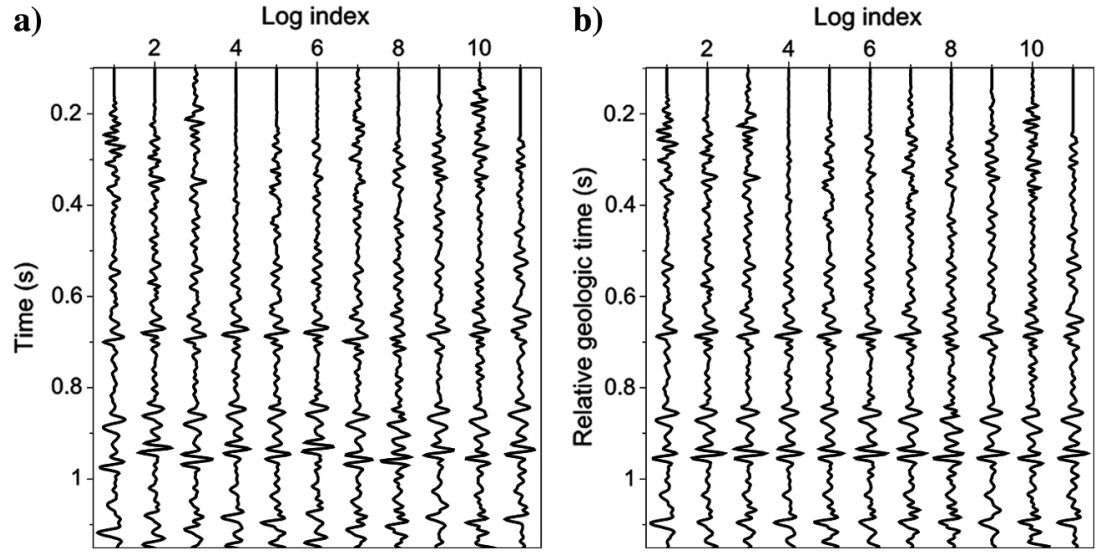

Figure 5. Real seismograms (nearest to the well locations) (a) before and (b) after flat-
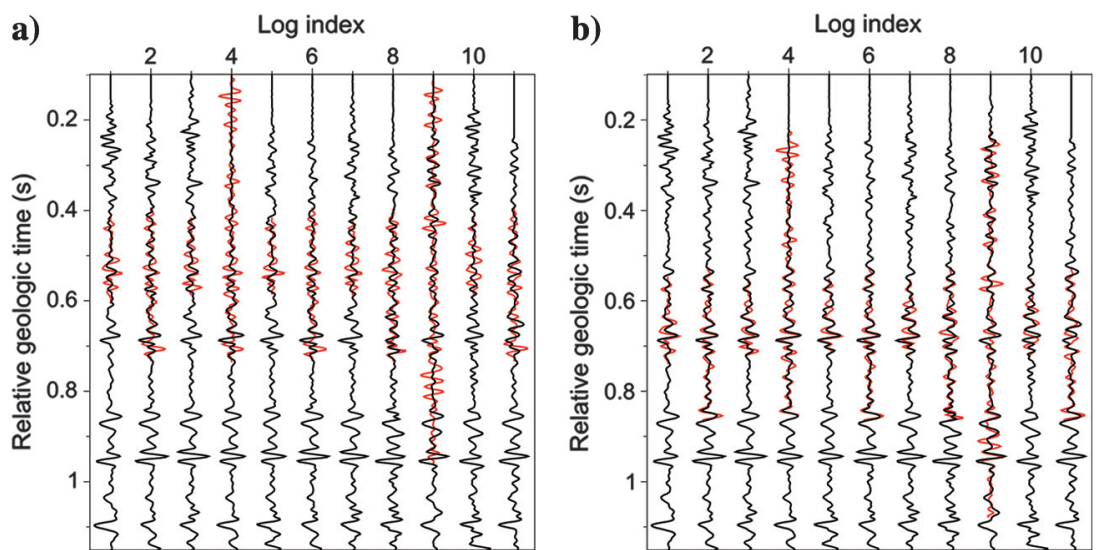

Figure 6. Flattened synthetic seismograms (red) and real seismograms (black) (a) before and (b) after alignment. 


$$
\min _{s_{u}\left(u_{f}\right)} \sum_{u_{f}} \frac{1}{N_{u_{f}}} \sum_{I}\left\|g\left(I, u_{g}\right)-f\left(I, u_{f}+s_{u}\left(u_{f}\right)\right)\right\|_{p},
$$

where $I$ is the index of the synthetic and real seismograms. The number of synthetic seismograms $N_{u_{f}}$ varies with RGT $u_{f}$ because some seismograms may be missing at some $u_{f}$ as shown in Figures $4 \mathrm{~b}$ and $6 \mathrm{a}$. Because the shifts $s_{u}\left(u_{f}\right)$ vary only vertically with $u_{f}$, we can efficiently perform minimization in equation 11 and obtain $s_{u}\left(u_{f}\right)$ using a $1 \mathrm{D}$ smooth DTW method (Compton and Hale, 2014).

Using the computed shifts $s_{u}\left(u_{f}\right)$, we match the flattened synthetic seismograms (red) with the flattened real seismograms (black) in RGT $u_{g}=u_{f}+s_{u}\left(u_{f}\right)$, as shown in Figure 6b. We can then use the flattening shifts $s_{g}(I, t)$ of the real seismograms to map these matched synthetic and real seismograms from RGT $u_{g}$ back to two-way time $t$ and obtain a simultaneous match of the multiple seismograms in $t$ as shown in Figure 7a. Next, we will discuss how to compute the time-depth functions $t(I, z)$ with the composite shifts $\left(s_{f}, s_{g}\right.$, and $\left.s_{u}\right)$ to directly match the synthetic seismograms with the real ones in the two-way time.

\section{Updating time-depth functions}

Computing time-depth functions $t(I, z)$ in this case is not as straightforward as in single well-seismic ties because we align the synthetic seismograms with real seismograms in RGT, instead of time. However, we can compute the time-depth functions $t(I, z)$ using time-RGT functions $t\left(I, u_{q}\right)$ and RGT-depth functions $u_{g}(I, z)$. The time-RGT function $t\left(I, u_{g}\right)$ can be computed from the flattening map $u_{g}(I, t)$ of real seismograms using an inverse interpolation method because $u_{g}(I, t)$ is a monotonic function of $t$ for each trace index $I$. Indeed, the RGT-depth functions $u_{g}(I, z)$ can be computed by

$$
u_{g}(I, z)=u_{f}(I, z)+s_{u}\left(u_{f}\right),
$$

where $u_{f}(I, z)=\tau(I, z)+s_{f}(I, \tau)$. Therefore, the time-depth functions $t(I, z)$ are computed by

$$
t(I, z)=t\left(I, u_{g}(I, z)\right)
$$
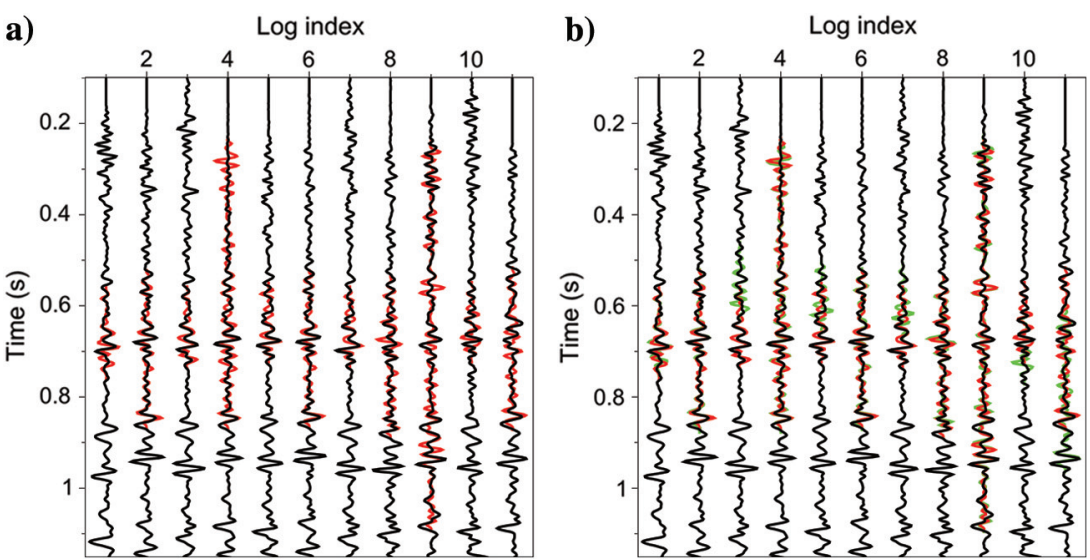

Figure 7. (a) The aligned synthetic (red) and real (black) seismograms in RGT (Figure 6 b) are mapped back to two-way time to obtain a simultaneous match of the multiple real and synthetic seismograms. These simultaneously matched synthetic seismograms (colored by red) are overlaid with those independently matched ones (colored by green) in (b).
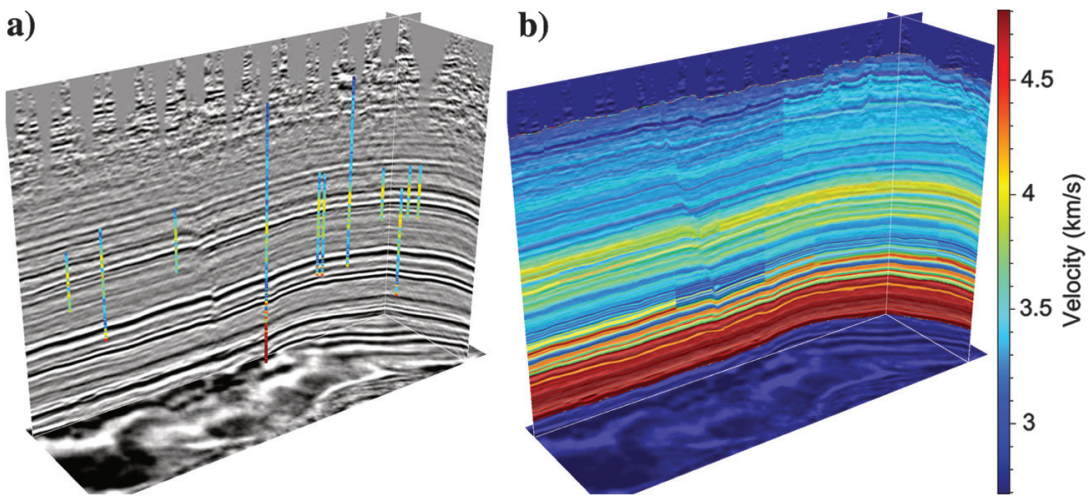

Figure 8. Velocity $\log$ s are simultaneously tied (a) to the seismic image using the timedepth functions computed from simultaneous multiple well-seismic ties. A 3D imageguided nearest neighbor interpolation (b) of these logs is laterally consistent along seismic reflectors. where $u_{g}(I, z)=\tau(I, z)+s_{f}(I, \tau)+s_{u}\left(\tau+s_{f}\right)$. As in the case of single well-seismic ties, we want to iteratively update the time-depth functions $t(I, z)$ using equation 13 . With the finally updated $t(I, z)$, we can directly compute the synthetic seismograms that match the real seismograms in two-way time, as shown in Figure 7a. In Figure 7b, we display all the aligned synthetic seismograms computed using single (green traces) and simultaneous (red traces) well-seismic tying methods together with the real seismic traces (black ones). We observe the alignments computed using the two methods are almost the same for the long seismograms but are significantly different for the short ones, especially the 3rd, 5th, 7th, 10th, and 11th seismograms.

With the finally updated time-depth functions $t(I, z)$, we tie all the velocity logs to the seismic image, as shown in Figure 8a. A 3D imageguided nearest neighbor interpolation (Figure 8a) of these tied velocity $\operatorname{logs}$ is laterally more continuous along seismic reflectors than the one in Figure $3 \mathrm{~b}$. This suggests that the simultaneous multiple well-seismic ties (Figure $8 b$ ) are more consistent than the single well-seismic ties (Figure 3a).

\section{DISCUSSION}

We have proposed to first laterally correlate the synthetic and real seismograms to obtain flattened seismograms. We then vertically matched the flattened synthetic seismograms with the flattened real seismograms to efficiently compute sistep approach should only be applied when the multaneous multiple well-seismic ties. This two- 
lateral correlations of the synthetic and real seismograms are trustworthy.

We believe that this methodology could also work with geologically complex settings (growth faults, rapid layer thickness variations, and late diagenetic units). However, our correlation of seismic traces and synthetic traces would likely yield inaccurate results in these cases. To improve seismic trace correlation, an alternative strategy could then be used to better exploit imaging information by flattening the whole seismic image (Lomask et al., 2006; Fomel, 2010; Wu and Zhong, 2012; Wu and Hale, 2015a, $2015 \mathrm{~b}, 2016$ ), instead of flattening only the seismograms extracted at well locations. To improve well correlations, one could also replace our DTW-based synthetic trace correlation by expert-based manual correlation or correlation using various logs or rules (Lallier et al., 2012, 2016).

In the case of rapidly varying structures in space, single wellseismic ties may be more reliable than the lateral correlations of synthetic seismograms or well logs. In such cases, it may help to use well-seismic ties to improve well-log correlation (or synthetic seismogram correlation), instead of using the latter to improve the former as proposed in this paper. The general idea in this case would be to first match well logs with the seismic image one by one using any single well-seismic tying method (Herrera and van der Baan, 2014; Herrera et al., 2014; Cheverry et al., 2015; Muñoz and Hale, 2015). These well logs could then be laterally correlated by the large-scale structure trend computed via seismic image flattening (Lomask et al., 2006; Fomel, 2010; Wu and Hale, 2015a, 2015b).

In this scenario, a seismic image would only provide a low-frequency structure trend for well-log correlation because of the uncertainties involved in seismic imaging process itself, in computing well-seismic ties, and in seismic image flattening. Still, such trends can be used to reduce ambiguities in automated stochastic well correlation problems, as proposed by Julio et al. (2012). One might use this low-frequency trend to first compute an initial correlation of the well logs and then use a well-log correlation method (Wheeler and Hale, 2014) to further tune the correlation to reflect high-frequency information that is measured in well logs but not in seismic data.

\section{CONCLUSIONS}

We have proposed a two-step correlation method to simultaneously tie multiple wells to a seismic image and obtain laterally consistent well-seismic ties. In the first step, we laterally correlate the synthetic seismograms and corresponding real seismograms separately and obtain flattened synthetic and real seismograms, in both of which events corresponding to the same geologic layers are horizontally aligned. We then vertically shift the flattened synthetic seismograms to match the flattened real ones using only vertically variant but horizontally invariant shifts, which is a $1 \mathrm{D}$ correlation problem. With this efficient two-step correlation method, we obtain the vertically and laterally optimized correlations of the well logs and the corresponding real seismograms. This procedure makes it possible to extrapolate well-log values using image-guided interpolation without notable artifacts. This result stems from the lateral consistency brought by the well correlation in the well-seismic ties.

In the first step, instead of flattening the whole seismic image, we only need to flatten or correlate the limited number of synthetic seismograms and corresponding real seismograms. This makes our simultaneous well-seismic tying method especially efficient. It took less than 5 min to simultaneously tie the 11 well $\operatorname{logs}$ to the seismic data in the example shown in this paper. However, the direct correlations of the synthetic or real seismograms may be unreliable in some cases, for example, with geologic layers disappearing between well locations. In these cases, a more reliable lateral correlation of the real seismograms may be computed by tracking seismic reflections between these seismograms at well locations. Further work may be worthwhile to introduce low-frequency structure trends that can be tracked from the seismic image, as prior constraints to reduce ambiguities in automatic well-log correlations. However, in this case, we need to first match well logs with corresponding real seismograms, probably using single well-seismic tying methods, and then use the large-scale structure trend tracked from the seismic image as a low-frequency control for well-log correlations.

\section{ACKNOWLEDGMENTS}

In much of the research described in this paper, we benefited greatly from discussions with D. Hale. We appreciate suggestions by J. K. Welford, R. Heggland, and two anonymous reviewers that led to significant revision of this paper. This research is supported by the sponsor companies of the Consortium Project on Seismic Inverse Methods for Complex Structures at the Colorado School of Mines. The Teapot Dome seismic and well data were provided by the Rocky Mountain Oilfield Testing Center.

\section{Nomenclature}

$\tau=$ two-way time of synthetic seismograms

$t \quad=$ two-way time of real seismograms

$I=$ index of seismograms

$f(I, \tau)=$ synthetic seismograms

$g(I, t) \quad=$ real seismograms

$s_{f}(I, \tau)=$ shifts flattening synthetic seismograms

$s_{g}(I, t)=$ shifts flattening real seismograms

$s_{u}\left(u_{f}\right)=1 \mathrm{D}$ shifts tying flattened seismograms

$u_{f}(I, \tau)=$ RGT of synthetic seismograms

$u_{g}(I, t)=$ RGT of real seismograms

$t\left(I, u_{g}\right)=$ time-RGT functions

$u_{g}(I, z)=$ RGT-depth functions

$t(I, z)=$ time-depth functions

\section{REFERENCES}

Anderson, K. R., and J. E. Gaby, 1983, Dynamic waveform matching: Information Sciences, 31, 221-242, doi: 10.1016/0020-0255(83)90054-3.

Anderson, T., 2009, History of geologic investigations and oil operations at Teapot Dome: Presented at the 2009 AAPG Annual Convention, Wyoming.

Cheverry, R., J. Edwards, and G. Caumon, 2015, Seismic-to-well tie using stochastic dynamic time warping: Presented at the 35th Gocad Meeting - 2015 RING Meeting, ASGA.

Compton, S., and D. Hale, 2014, Estimating $V_{\mathrm{P}} / V_{\mathrm{S}}$ ratios using smooth dynamic image warping: Geophysics, 79 , no. 6 , V201-V215, doi: 10 $.1190 /$ geo2014-0022.1.

Cubizolle, F., T. Valding, S. Lacaze, and F. Pauget, 2015, Global method for seismic-well tie based on real time synthetic model: 85th Annual International Meeting, SEG, Expanded Abstracts, 1776-1781.

Fehmers, G. C., and C. F. Höcker, 2003, Fast structural interpretation with structure-oriented filtering: Geophysics, 68, 1286-1293, doi: 10.1190/1 .1598121.

Fomel, S., 2010, Predictive painting of 3D seismic volumes: Geophysics, 75 , no. 4, A25-A30, doi: 10.1190/1.3453847.

Hale, D., 2009, Image-guided blended neighbor interpolation of scattered data: 79th Annual International Meeting, SEG, Expanded Abstracts, $1127-1131$. 
Hale, D., 2010a, Image-guided 3D interpolation of borehole data: CWP Report 656

Hale, D., 2010b, Image-guided 3D interpolation of borehole data: 80th Annual International Meeting, SEG, Expanded Abstracts, 1266-1270.

Hale, D., 2013, Dynamic warping of seismic images: Geophysics, 78, no. 2, S105-S115, doi: 10.1190/geo2012-0327.1.

Herrera, R. H., S. Fomel, and M. van der Baan, 2014, Automatic approaches for seismic to well tying: Interpretation, 2, no. 2, SD9-SD17, doi: 10 $.1190 /$ INT-2013-0130.1.

Herrera, R. H., and M. van der Baan, 2014, A semiautomatic method to tie well logs to seismic data: Geophysics, 79, no. 3, V47-V54, doi: 10.1190/ geo2013-0248.1.

Julio, C., F. Lallier, and G. Caumon, 2012, Accounting for seismic trends in stochastic well correlation, in Geostatistics: Springer, Quantitative Geology and Geostatistics 17, 251-262.

Lallier, F., C. Antoine, J. Charreau, G. Caumon, and J. Ruiu, 2013, Management of ambiguities in magnetostratigraphic correlation: Earth and Planetary Science Letters, 371-372, 26-36, doi: 10.1016/j.epsl.2013.04.019.

Lallier, F., G. Caumon, J. Borgomano, S. Viseur, F. Fournier, C. Antoine, and T. Gentilhomme, 2012, Relevance of the stochastic stratigraphic well correlation approach for the study of complex carbonate settings: Application to the Malampaya buildup (Offshore Palawan, Philippines): Geological Society, Special Publication, 370, 265-275, doi: 10.1144/ SP370.12.

Lallier, F., G. Caumon, J. Borgomano, S. Viseur, J.-J. Royer, and C. Antoine, 2016, Uncertainty assessment in the stratigraphic well correlation of a carbonate ramp: Method and application to the Beausset Basin, SE France: Comptes Rendus Geoscience.

Lomask, J., A. Guitton, S. Fomel, J. Claerbout, and A. A. Valenciano, 2006 Flattening without picking: Geophysics, 71, no. 4, P13-P20, doi: 10 $.1190 / 1.2210848$.

Muñoz, A., and D. Hale, 2012, Automatically tying well logs to seismic data: CWP-725.

Muñoz, A., and D. Hale, 2014, Automatic simultaneous multiple-well ties: 84th Annual International Meeting, SEG, Expanded Abstracts, 1512-1516.

Muñoz, A., and D. Hale, 2015, Automatic simultaneous multiple well ties: Geophysics, 80, no. 5, IM45-IM51, doi: 10.1190/geo2014-0449.1.

Sakoe, H., and S. Chiba, 1978, Dynamic programming algorithm optimization for spoken word recognition: IEEE Transactions on Acoustics, Speech, and Signal Processing, 26, 43-49, doi: 10.1109/TASSP.1978 .1163055 .
Sheriff, R. E., and L. P. Geldart, 1995, Exploration seismology: Cambridge University Press.

Smith, T., and M. Waterman, 1980, New stratigraphic correlation techniques: Journal of Geology (United States), 88, 451-457, doi: 10 $.1086 / 628528$.

Stark, T. J., 2004, Relative geologic time (age) volumes - Relating every seismic sample to a geologically reasonable horizon: The Leading Edge, 23, 928-932, doi: 10.1190/1.1803505.

Van Vliet, L. J., and P. W. Verbeek, 1995, Estimators for orientation and anisotropy in digitized images: Proceedings of the First Annual Conference of the Advanced School for Computing and Imaging ASCI'95, Heijen (The Netherlands), 442-450.

Walden, A. T., and R. E. White, 1998, Seismic wavelet estimation: a frequency domain solution to a geophysical noisy input-output problem: IEEE Transactions on Geoscience and Remote Sensing, 36, 287-297, doi: $10.1109 / 36.655337$.

Wheeler, L., and D. Hale, 2014, Simultaneous correlation of multiple well logs: 84th Annual International Meeting, SEG, Expanded Abstracts, 618622

White, R., 1980, Partial coherence matching of synthetic seismograms with seismic traces: Geophysical Prospecting, 28, 333-358, doi: 10.1111/j .1365-2478.1980.tb01230.x.

White, R., 1997, The accuracy of well ties: Practical procedures and examples: 67th Annual International Meeting, SEG, Expanded Abstracts, 816819.

White, R., and R. Simm, 2003, Tutorial: Good practice in well ties: First Break, 21, 75-83.

Wu, X., and D. Hale, 2015a, 3D seismic image processing for unconformities: Geophysics, 80, no. 2, IM35-IM44, doi: 10.1190/geo2014-0323.1.

Wu, X., and D. Hale, 2015b, Horizon volumes with interpreted constraints: Geophysics, 80, no. 2, IM21-IM33, doi: 10.1190/geo2014-0212.1.

$\mathrm{Wu}$, X., and D. Hale, 2016, Automatically interpreting all faults, unconformities, and horizons from 3D seismic images: Interpretation, 4, no. 2, T227T237, doi: 10.1190/INT-2015-0160.1.

Wu, X., and G. Zhong, 2012, Generating a relative geologic time volume by $3 \mathrm{D}$ graph-cut phase unwrapping method with horizon and unconformity constraints: Geophysics, 77, no. 4, O21-O34, doi: 10.1190/ geo2011-0351.1.

Ziolkowski, A., J. R. Underhill, and R. G. K. Johnston, 1998, Wavelets, well ties, and the search for subtle stratigraphic traps: Geophysics, 63, 297313, doi: 10.1190/1.1444324. 